
ISSN 1813-548X

\title{
Effet létal de l'extrait aqueux de l'algue brune marine (Cystoseira tamariscifolia) sur la souris et sur les cellules tumorales du myélome murin
}

\section{Zineb SOUHAILI ${ }^{*}$, Hicham MOHAMMADI ${ }^{1}$, Noureddine HABTI ${ }^{2}$ et Mohamed FAID ${ }^{3}$}

'Laboratoire de Biochimie, Faculté de Médecine et de Pharmacie, 19, rue Tarik bnou Ziad, Casablanca, Maroc

2Laboratoire d'Hématologie, Faculté de Médecine et de Pharmacie, Casablanca, Maroc

${ }^{3}$ Laboratoire de biotechnologie alimentaire, I.A.V Hassan II, Rabat, Maroc

*Correspondance, courriel : z.souhaili@gmail.com

\section{Résumé}

L'espèce Cystoseira tamariscifolia est une algue brune marine qui constitue une biomasse algale importante sur la côte atlantique Marocaine. L'une des perspectives de notre étude sur cette algue était sa valorisation nutritionnelle en vue d'une exploitation économique comme valeur ajoutée aussi bien en alimentation humaine qu'animale à l'instar de plusieurs algues marines comestibles. Pour cette raison, nous avons été amené à étudier la toxicité aigue de cette espèce et déterminer sa DL50 qui a été réalisée selon la méthode de Spearman-Karber, en injectant par voie intra péritonéale à un groupe de 36 souris Swiss albinos, répartis en 6 lots de six souris chacun, une dose de $0.5 \mathrm{~mL}$ de l'extrait aqueux de l'algue à une concentration donnée, En parallèle une étude de l'effet cytotoxique de l'algue sur les cellules tumorales du myélome murin P3/X63-Ag8.653 a été réalisée en utilisant l'extrait aqueux de l'algue à différentes concentrations. Les résultats obtenus ont montré un net effet toxique de l'algue sur les souris avec une $\mathrm{DL}_{50}$ 
$738.61 \pm 34 \mu \mathrm{g}$ d'algue $/ \mathrm{g}$ de poids corporel. Nous avons aussi observé un effet cytotoxique de l'algue sur les cellules myélomateuses proportionnel aux concentrations utilisées.

Mots-clés : Algue brune marine, Cystoseiracea, extraction, toxicité, cytotoxicité

\section{Abstract \\ Lethal effect of the aqueous extract of the brown marine algae (Cystoseira tamariscifolia) on the mouse and the myelom cells}

Study of the acute toxicity of Cystoseira tamariscifolia a brown algae widely speared along the Moroccan Atlantic coast, was carried out on Swiss Albinos mice, the LD 50 was determined according to the method of Spearman-Karber by injecting $0.5 \mathrm{ml}$ of the aqueous algal extract to 36 mice organized in lots of 6 animals each for 6 concentrations and experiments were carried out in triplicates. Amounts of $0.5 \mathrm{ml}$ of each concentration of the extract were injected to the mice intraperitonially. The study of the cytotoxic effect was carried out on the myelom cells P3/X63-Ag8.653, using different concentrations of the extract. Results showed a toxic effect of the algae extract on mice with a LD 50 of $738.61 \pm 34 \mu \mathrm{g}$ of the algae/g of body weight. The cytotoxic effect was also detected on the myelom cells; it's proportional to the used concentrations.

Keywords : Brown marine algae, Cystoseiracea, extraction, toxicity, cytotoxicity.

\section{Introduction}

Les algues brunes marines sont des algues largement répandues à travers le monde, ceci revient à leur faculté d'adaptation via leur reproduction et leur réponse à des conditions écologiques variées. Ceci suppose que les algues brunes sécrètent des substances chimiques de défense, contre les multiples dangers auxquels elles sont exposées (prédateurs mobiles, et micro-organismes envahisseurs) [1]. Ainsi 
plusieurs équipes de recherche se sont intéressées aux différents aspects de défense de ces algues, surtout dans un but de recherche de substances naturelles à activités antimicrobiennes [2-7] ou encore à pouvoir toxique ou antitumoral [8-11].

Cystoseira, est un genre d'algue brune marine appartenant à la classe des Phéophycées; ordre des fucales. II comprend 50 à 55 espèces dont Cystoseira tamariscifolia qui est appelée aussi Cystoseira erricoides [12, 13], c'est une plante robuste de 10 à $50 \mathrm{~cm}$ de long, de couleur vert olive, produisant une sensation rude au toucher, c'est une espèce largement répandue sur la côte atlantique Marocaine [14] qui a suscité peu l'intérêt des chercheurs par comparaison à d'autres espèces d'algues brunes marines tel que Bifurcaria bifurcata $[8,10]$ ou Cystoseira crinita $[4,5,7]$.

Les seules études effectuées sur Cystoseira tamariscifolia sont réalisées par Bennamara et al. [15] portant sur une étude chimique d'un principe actif purifié (un méroditerpène) et son effet antimicrobien sur Escherichia coli et Botrytis cinerea et les recherches effectuées par notre équipe portant sur l'activité antifongique et antimycotoxines de cette algue [16]. Toutefois, l'étude de l'effet toxique et cytotoxique de Cystoseira tamariscifolia n'a jamais été réalisée.

Notre étude a été réalisée dans un but de valorisation nutritionnelle de l'espèce Cystoseira tamariscifolia en vue d'une exploitation économique comme valeur ajoutée aussi bien en alimentation humaine qu'animale à l'instar de plusieurs algues marines comestibles. Pour cela une étude de son effet toxique et cytotoxique s'est avérée nécessaire.

L'étude de la toxicité aigue de l'algue et sa DL50 a été réalisée sur son extrait aqueux par injection intrapéritonéal chez la souris Swiss albinos. L'effet cytotoxique a été réalisé sur des myélomes de souris en culture. 


\section{Matériel et méthodes}

\section{2-1. Récolte}

La récolte de l'algue Cystoseira tamariscifolia a été effectuée entre le printemps et l'été, au niveau de la région de Témara (Oued Ykem) à basse mer. Le poids de la récolte a été estimé à $10 \mathrm{~kg}$, le matériel frais est mis dans des sacs en plastique il a été ensuite rincé à l'eau douce plusieurs fois pour éliminer les sels minéraux et les contaminants avant d'être finement coupé et séché pendant $48 \mathrm{~h}$ à une température ambiante d'environ $35^{\circ} \mathrm{C}$ à l'abri de la lumière solaire dans un endroit ombragé exposé au courant d'air sur un support de grille en bois, ensuite l'algue sèche a été conservée dans des sachets en papier fermés à l'abri de l'humidité et de la lumière.

\section{2-2. Préparation de l'extrait}

$5 \mathrm{~g}$ de l'algue sèche a été broyée dans un mortier avec $100 \mathrm{~mL}$ d'eau distillée chaude la solution obtenue est mélangée à l'aide d'un vortex à 3 dimensions pendant une nuit et centrifugée ensuite à $3000 \mathrm{~g}$ pendant 30 min, le surnageant récupéré subit une filtration stérilisante grâce à une membrane millipore $0.22 \mu \mathrm{m}$. Le filtrat est aliquoté dans des tubes eppendorf et immédiatement congelé à $-20^{\circ} \mathrm{C}$ à l'abri de la lumière.

\section{2-3. Test de toxicité aigue et DL50}

\section{2-3.1. Détermination de la DL50}

Pour permettre une meilleure biodisponibilité du(es) principe(s) actif(s), le test de toxicité aigue a été réalisé par injection intra péritonéale de l'extrait algal à un groupe de 36 souris Swiss albinos de poids allant de 18 à 20g, élevées à l'animalerie du centre d'expérimentation de l'institut pasteur du Maroc. Les animaux ont été répartis en 6 lots, de six souris chacun, chaque lot de souris a reçu une injection de $0.5 \mathrm{~mL}$ de l'extrait aqueux de l'algue à une concentration déterminée. Les concentrations étaient choisies de telle manière à mieux encadrer la DL50, on part d'une concentration à $100 \%$ de mortalité à une concentration à $0 \%$ de 
mortalité [17]. Les concentrations utilisées étaient de $5 \%, 4.16 \%, 3.47$ $\%, 2.89 \%, 2.41 \%$ et $2.01 \%$ obtenues par dilutions successives, selon un facteur de dilution de 1.2 à partir d'une solution mère de l'extrait aqueux à $5 \mathrm{~g} / 100 \mathrm{~mL}$, préparée comme décrit précédemment -chaque concentration a été testée 3 fois-. On enregistre les souris mortes pendant $48 \mathrm{~h}$ et on calcule la dose létale 50 par la méthode de SpearmanKarber [17].

\section{2-4. Toxicité cellulaire}

La lignée cellulaire utilisée est la lignée myélomateuse P3/X63-Ag8.653, issue de la souris Balb/ $C$ offerte par l'établissement de transfusion sanguine d'acquitaine-Bordeaux. Ce sont des cellules tumorales qui se multiplient indéfinement dans le temps en échappant à l'apoptose.

Les cellules ont été cultivées dans des plaques de culture de six puits (Corning, EtatsUnis) à une concentration de $0,1.10^{6}$ cellules $/ \mathrm{ml}$ dans du milieu RPMI 1640 (Eurobio, France) contenant du Sérum de Veau Fœtal (SVF, Eurobio, France) à $15 \%$, de la L- Glutamine (Eurobio, France) à 2 $\mathrm{mM}$, de la pénicilline (Eurobio, France) à $100 \mathrm{Ul} / \mathrm{mL}$ et de la streptomycine (Eurobio, France) à 100 $\mathrm{gg} / \mathrm{mL}$ L'incubation en atmosphère humide à $37^{\circ} \mathrm{C}$ et à $5 \% \quad \mathrm{CO}_{2}$ dure 12 jours. Les cellules ne sont utilisées dans les tests que quand elles atteignent la phase exponentielle de croissance, soit une concentration de cellules allant de 0.5 à $0.7 .10^{6}$ cellules $/ \mathrm{mL}$. Trois concentrations de l'échantillon ont été étudiées $1 \mathrm{mg} / \mathrm{mL}, 2 \mathrm{mg} / \mathrm{mL}$ et $5 \mathrm{mg} / \mathrm{mL}$. A partir de l'extrait aqueux de l'algue à $5 \mathrm{~g} / 100 \mathrm{~mL}$, on prélève respectivement $100 \mu \mathrm{L}, 200 \mu \mathrm{l}$ et $500 \mu \mathrm{L}$ qu'on met en présence du milieu RPMl avec les cellules du myélome murin de tel manière à obtenir un volume final de $5 \mathrm{~mL}$. Ensuite, la boite à 6 puits dans laquelle on a mis les échantillons (cellules de myélome en culture + extrait aqueux) et le témoin (contient les cellules de myélome en culture + eau distillée stérile), est étiquetée et incubée à $37^{\circ} \mathrm{C}$. Les cellules ont été quotidiennement observées au microscope optique à statif inversé (Hund, wetzlar). La concentration cellulaire a été évaluée tous les 3 jours par numération cellulaire à l'aide d'une cellule de Malassez selon le principe d'exclusion au bleu trypan. Tous les tests ont été réalisés 3 fois. 


\section{2-5. Analyse statistique}

L'analyse statistique de l'effet algue sur les cultures de cellules de myélome murin traitées par rapport aux cultures témoins a été effectuée par comparaison des résultats de la viabilité cellulaire en utilisant le test de X2 à un seuil de probabilité de 0.05 .

L'effet concentration de l'extrait algal sur les cultures cellulaires a été analysé par comparaison des moyennes de la viabilité cellulaire par le test ANOVA à un seuil de probabilité de 0.05 .

\section{Résultats et discussion}

\section{3-1. Test de toxicité aigue sur souris}

\section{3-1-1. Détermination de la $D L_{50}$}

Les résultats de la détermination de la dose létale 50 (DL 50) moyenne effectuée à 3 reprises sur des souris ayant reçu $0.5 \mathrm{ml}$ de l'extrait algal à différentes concentrations par voie intrapéritoniale sont représentés dans le Tableau 1. La DL50 moyenne calculée par la formule de SpearmanKarber est : $738.61 \pm 34.03 \mu \mathrm{g}$ d'algue $/ \mathrm{g}$ de souris, donc il suffit d'une dose de $738.61 \mu \mathrm{g}$ d'algue $/ \mathrm{g}$ pour tuer $50 \%$ de l'effectif total. Ceci suppose la présence de toxines dans l'extrait algal dont la nature reste à déterminer.

Pour chaque concentration de l'extrait aqueux de l'algue le test a été réalisé sur trois lots différents de 6 souris chacun. Pour une concentration de $5 \mathrm{~g} / 100 \mathrm{ml}$ on obtient $100 \%$ de mortalité des souris et pour une concentration de $2.1 \mathrm{~g} / 100 \mathrm{ml}$ on obtient $0 \%$ de mortalité, entre les deux on obtient un taux de mortalité proportionnel à la concentration. 
Tableau 1: Nombre de mortalité des souris après injection intrapéritoniale de $0.5 \mathrm{~mL}$ de l'extrait aqueux de l'algue à différentes concentrations

\begin{tabular}{cccc}
\hline \multirow{2}{*}{$\begin{array}{c}\text { Dose en } \mu \mathrm{g} / \mathrm{g} \\
\text { (souris) }\end{array}$} & \multicolumn{3}{c}{$\begin{array}{c}\text { Nombre de souris mortes par lots } \\
\text { pour chaque test }\end{array}$} \\
\cline { 2 - 4 } & Test 1 & Test 2 & Test 3 \\
\hline C1 $=1250$ & $6 / 6$ & $6 / 6$ & $6 / 6$ \\
C2 $=1041.66$ & $5 / 6$ & $5 / 6$ & $5 / 6$ \\
C3 $=868.05$ & $3 / 6$ & $5 / 6$ & $4 / 6$ \\
C4 $=723.27$ & $3 / 6$ & $4 / 6$ & $3 / 6$ \\
C5 $=602.81$ & $2 / 6$ & $2 / 6$ & $2 / 6$ \\
C6 $=502.34$ & $0 / 6$ & $0 / 6$ & $0 / 6$ \\
DL50 & DL1 $=768.61$ & DL2 $=701.63$ & DL3 $=745.60$ \\
& \multicolumn{3}{c}{} \\
\hline
\end{tabular}

\section{3-2. Test de cytotoxicité}

Les cellules du myélome murin ont été mises en culture à la concentration de $0.110^{6}$ cellules $/ \mathrm{mL}$. Les résultats de l'effet de l'extrait aqueux de l'algue à différentes concentrations sur la viabilité des cellules du myélome murin en fonction du temps sont présentés dans le Tableau 2 et illustrés par la Figure 1. Le test de cytotoxicité a montré une diminution de la concentration des cellules viables dans le milieu de culture additionné de l'extrait de l'algue à différentes concentrations: $1 \mathrm{mg} / \mathrm{mL}, 2 \mathrm{mg} / \mathrm{mL}$ et $5 \mathrm{mg} / \mathrm{mL}$ par rapport aux cultures témoins sans extrait algal. En effet, après comptage des cellules par la cellule de malassez, on constate une diminution significative très nette des cellules viables après culture en présence de l'extrait algal par rapport aux cultures témoins aussi bien pour le jour J3, J6, J9 que J12. Ceci d'une 
part, d'une autre part, on constate que l'effet létal de l'extrait algal sur les cellules de myélome est proportionnel à la concentration de ce dernier dans le milieu de culture : la comparaison des cellules viables dans les cultures traités par les trois concentrations algales a montré une différence significative entre les concentrations $1 \mathrm{mg} / \mathrm{mL}$ et $5 \mathrm{mg} / \mathrm{mL}$ et entre les concentrations $2 \mathrm{mg} / \mathrm{mL}$ et $5 \mathrm{mg} / \mathrm{mL}$ pour les jours $\mathrm{J} 3, \mathrm{~J} 6$, J9 et $\mathrm{J} 12$, par contre la différence de la viabilité pour les concentrations $1 \mathrm{mg} / \mathrm{mL}$ et $2 \mathrm{mg} / \mathrm{mL}$ ne sont pas significatives pour le jour J6.

Tableau 2 : Nombre moyen de cellules myélomateuses $\left(\times 10^{3}\right) / \mathrm{mL}$ en fonction du temps après leur culture en présence de différentes concentrations de l'extrait aqueux de l'algue

\begin{tabular}{lccccc}
\hline Jours & J0 & J 3 & J 6 & J 9 & J12 \\
Extraits algaux & & & & & \\
\hline Témoin & 100 & $740 \pm 45^{\mathrm{a}}$ & $1000 \pm 25^{\mathrm{a}}$ & $700 \pm 20^{\mathrm{a}}$ & $300 \pm 8^{\mathrm{a}}$ \\
Extrait $(1 \mathrm{mg} / \mathrm{mL})$ & 100 & $340 \pm 35^{\mathrm{b}}$ & $318 \pm 6^{\mathrm{bc}}$ & $350 \pm 27^{\mathrm{b}}$ & $70 \pm 3^{\mathrm{b}}$ \\
Extrait $(2 \mathrm{mg} / \mathrm{mL})$ & 100 & $280 \pm 16^{\mathrm{c}}$ & $318 \pm 3^{\mathrm{bc}}$ & $250 \pm 10^{\mathrm{c}}$ & $60 \pm 2.7^{\mathrm{c}}$ \\
Extrait $(5 \mathrm{mg} / \mathrm{mL})$ & 100 & $100 \pm 7^{\mathrm{d}}$ & $194 \pm 1.5^{\mathrm{d}}$ & $120 \pm 4^{\mathrm{d}}$ & $20 \pm 1.85^{\mathrm{d}}$ \\
& & & & $\mathrm{P}<0.05$ & $\mathrm{P}<0.05$ \\
Niveau de signification & $\mathrm{P}<0.05$ & $\mathrm{P}<0.05$ & & \\
\hline
\end{tabular}

$\boldsymbol{a}, \boldsymbol{b}, \boldsymbol{c}$ et $\boldsymbol{d}$ correspondent aux différences significatives entre les moyennes au niveau d'une même colonne

Ce résultat laisse penser à un effet antitumoral de l'extrait algal, ceci concorde avec les travaux de Kotaké et al.[10] qui ont montré que la fucoxanthine qui est un caroténoide extraite des algues brunes a un important pouvoir antitumoral sur les cellules prostatiques cancéreuses 
chez l'Homme. De même, l'équipe de Ayyad et al. $[9,18]$ a pu purifier des algues brunes marine Sargassium crispum et Cystoseira myrica un méroditerpène qui possède un pouvoir cytotoxique, ce principe actif été aussi purifié de l'algue brune Cystoseira baccata par l'équipe de Mokrini et al. [19]. Par ailleurs, des études similaires effectuées sur l'algue brune Bifurcaria bifurcata ont montré que cette dernière contient des composés dotés d'importants pouvoirs cytotoxiques sur plusieurs lignées de cellules tumorales humaines en culture [8].

La représentation graphique de la viabilité des cellules du myélome murin dans les quatre milieux de culture (témoin et traité) en fonction du temps est illustrée par le graphe ci-dessous :

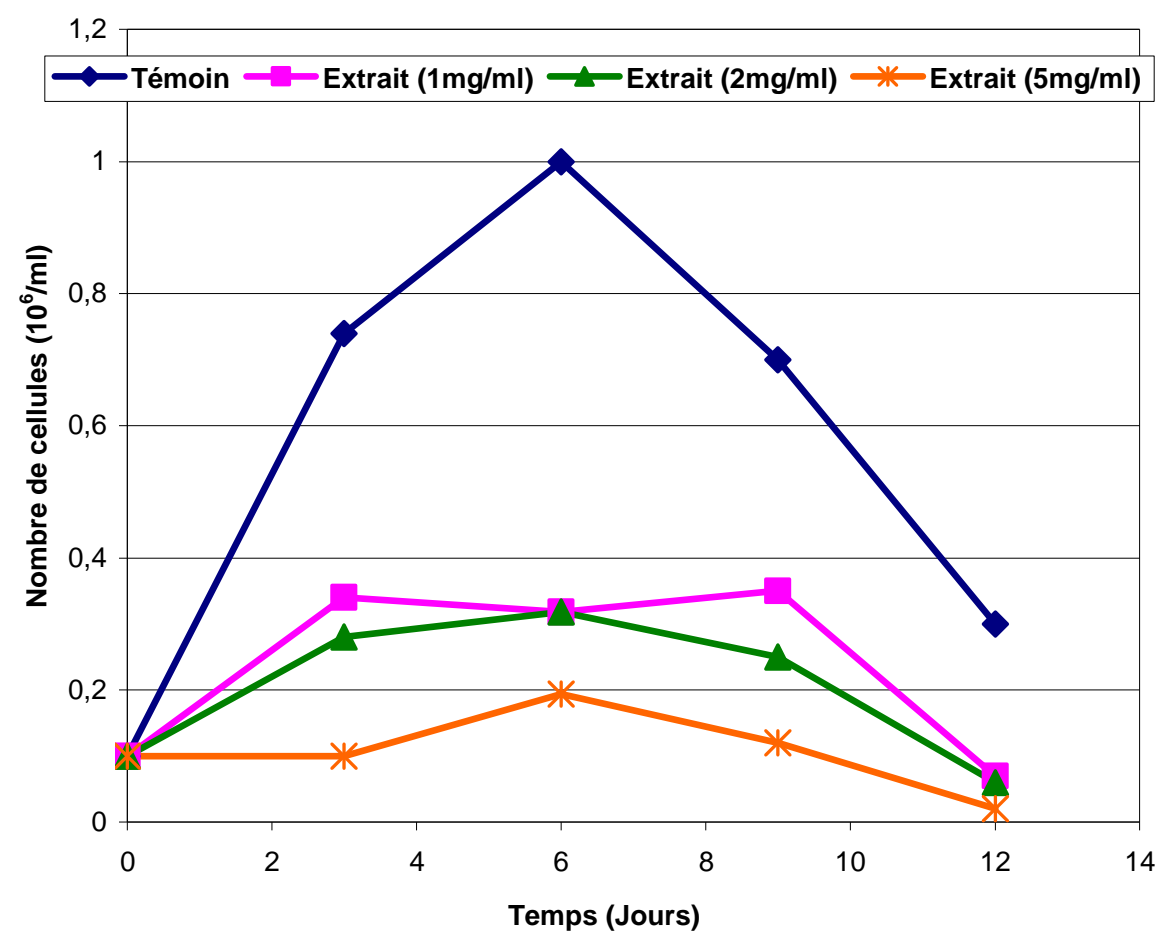

Figure 1: Viabilité en fonction du temps des cellules myélomateuses en culture en présence de l'extrait algal à différentes concentrations 


\section{Conclusion}

D'après les résultats obtenus on peut conclure que l'algue Cystoseira tamariscifolia présente une toxicité nette sur la souris et donc il serait impossible de l'exploiter comme valeur ajoutée en alimentation humaine ou animale, toutefois sa cytotoxicité sur les cellules tumorales $d u$ myelome murin restent encourageants pour une étude antitumorale in vivo et la recherche d'un éventuel principe actif à caractère anticancéreux.

\section{Références}

[1] - G. CULIOLI, C. VINCENT-CHABROL, L. PIOVETTI, R.VALLS, Cryptogamie Algologie, 19 (1998) 257-258

[2] - J.F. BIARD, J.F. VERBIST, Y. LET OURNEUX, R. FLOCH, Planta Med. 40 (1980) 288-294

[3] - P. LEBERTON, Rev. Ecol. Terre vie, 36 (1982) 4

[4] - A. PRAUD, R.VALLS, L. PIOVETTI, B. BANAIGS, G. MALYNGAMIDE, Tetrahedron Letters, 34 (1993) 5437-5440

[5] - A. PRAUD, R.VALLS, L. PIOVETTI, B. BANAIGS, J. BENAIM, Photochemistry, 40 (1995) 495-500

[6] - G. OZDEMIR, Z. HORZUM, A.SUKATAR, N. KARABAYYAVASOGLU, Pharmaceutical Biology (Formerly International Journal of Pharmacognosy), 44(3) (2006) 183-188(6)

[7] - Z. KAMENARSKAA, N. FUNDA, F.N. YALÇN, T. ERSOZ, I. CALIS, Naturforsch. , 57 (2002) 584-590

[8] - A. DIGUARDIA, R. VALLS, V. MESGUICHE, J.M. BRUNEL, G. CULIOLI., Tetrahedron Letters, 40 (1999) 8359-8360

[9] - S.E. AYYAD, M.O. SALMA, A.H.MOKHTAR, A.F. ANTER, Biol Chim. Fram, 140 (2001) 155-159

[10] - N. KOTAKE, M. KUSHIRO, H. ZHANG, T. SUGAWARA, K. MIYASHITA, A. NAGAO, J. Nutr., 131 (12) (2001) 3303-6 
[11] - G. CULIOLI, M. DAOUDI, A. ORTALO-MAGNE, R. VALLS, L. PIOVETTI, Phytochemistry, 57 (2001) 529-35

[12] - P. DANGEARD, Le botaniste, 34 (1949) 89-198

[13] - M. ROBERTS , Phycol. Bull. , 3 (1967) 245-366

[14] - P. GAYRAL, Bull. Soc. Sc. Nat. et Phys. Maroc, 527p (1958)

[15] - A. BENNAMARA, A. ABOURRICH, M. BERRADA, M. CHARROUF, N. CHAIB, M. BOUDOUMA, M. GARNEAU, Phytochemistry , 52 (1999) 37-40

[16] - Z. SOUHAILI, M. LAGZOULI, M. FAID, K. FELLAT-ZARROUCK, Afr. J .biotech., 3(1) (2004) 71-75

[17] - JA. GENÉ, and A. ROBLES, Rev. Med. Hosp. Nal. Niños. Costa Rica, 1 (1989) 35-40

[18] - :AYYAD, O.B. ABDEL-HALIM, W.T. SHIER , T.R. HOYE, Z. Naturforsch [C], 58(1-2) (2003) 33-38

[19] - R. MOKRINI, M. BEN MESAOUD, M. DAOUDI, C. HELLIO, J.P. MARÉCHAL, M. EL HATTAB, A. ORTALO-MAGNÉ, L. PIOVETTI, G. CULIOLI, J. Nat. Prod, 71 (11) (2008) 1806-1811 